\title{
Cerebral Venous Thrombosis as a Cause of Acute Headache
}

\author{
Michael Grover, DO
}

Most patients presenting to the family practice office or emergency department (ED) have benign causes for their headache symptoms. However, it is vitally important to recognize high-risk clinical features that could represent intracranial pathology. Cerebral venous (CVT) and dural sinus thrombosis is a diagnosis made with increasing regularity because of improved awareness of the condition and the availability of noninvasive testing modalities.

\section{Case Presentation}

A 37-year-old man with a past medical history of hypertension and seizure disorder presented to the ED with complaint of severe headache with speech and vision difficulties. He had a 3-week history of cold symptoms and antecedent strep throat treated with penicillin 1 week before presentation to the ED. He had experienced mild to moderately severe headaches that he had treated with ibuprofen for the prior 5 days. The night before presentation, the patient complained of a much-increased headache in the left temporal area. This was severe, much more intense then any previous headache. $\mathrm{He}$ noted associated difficulty with conversations in that he was making frequent word errors and having difficulty finding the correct word to say. The next morning, the day of admission, patient could not read the newspaper well, finding it difficult to understand the words on the page. The patient's wife, who brought the patient to the emergency department, also noted the retrieval errors.

The patient's hypertension and epilepsy had been well controlled medically. He had no history of tobacco, alcohol, or drug use and had no significant family history. Physical examination revealed normal vital signs, except mild hypertension at

Submitted, revised, 29 October 2003.

From the Department of Family Medicine, Loma Linda University, Loma Linda, California. Address correspondence to Michael Grover, DO, Department of Family Medicine, Mayo Clinic in Scottsdale, 13737 N. 92nd St., Scottsdale, AZ 85260 (E-mail: grover.michael@mayo.edu).
144/90 mm Hg. Patient was afebrile, alert, and oriented. Head, eyes, ears, nose, and throat examinations were unremarkable, with fundi having no papilledema. Cranial nerves were intact, and strength, sensation, reflexes, Babinski reflex, and results of gait testing were normal. No focal deficits were noted except difficulty naming objects. Abstract thinking was intact and spontaneous language use while providing the history was fluent.

Laboratory testing showed blood chemistries and complete blood count to be normal. Prothrombin time and partial thromboplastin time were not increased. Computed tomography (CT) of the head showed a left temporal hemorrhagic infarction. No subdural hematoma or midline shift was appreciated. After spinal fluid and blood cultures were obtained, the patient was started on intravenous antibiotics (because of the concern over the possibility of associated mastoiditis or meningitis) and admitted to the intensive care unit. The next day the patient underwent magnetic resonance imaging (MRI) of the head, which again demonstrated the left temporal hemorrhage but did not reveal any evidence of mastoiditis. He also had MR angiogram (MRA) and MR venogram (MRV) tests. The MRA was negative for aneurism or other abnormalities. The MRV demonstrated occlusion of the left transverse sinus, sigmoid sinus, and the visualized portion of the internal jugular vein. The diagnosis of cerebral venous sinus thrombosis was made. Intravenous heparin therapy was therefore initiated.

The patient was treated in hospital for a total of 8 days. His neurologic signs and symptoms slowly improved over the initial 72 hours. Spinal fluid cultures and blood cultures were negative. Earnose-throat consultants felt that mastoiditis or other infectious causes (encephalitis, meningitis) were ruled out. The patient was neurologically intact at discharge except for mild exertional headache and sent home on a 6-month course of warfarin for anticoagulation. After warfarin treatment was completed, he was tested for an underlying hypercoagulable state. Factor V Leiden mutation 
was discovered, and life-long warfarin therapy was recommended.

\section{Discussion}

CVT results from the occlusion of a venous sinus by a partially obstructing thrombus or an extrinsic compression that eventually progresses to completely occlude it. ${ }^{1}$ Once the vein is occluded, the thrombus may extend to veins draining into the sinus. The resulting venous congestion can lead to regional ischemia and infarction in the cerebral cortex. The most frequently occluded venous sinuses are the superior sagittal, the left transverse, and the right transverse. ${ }^{2}$

Patients with CVT present differently from those with conventional arterial stroke. ${ }^{3}$ Clinical presentations of CVT are variable, making it a diagnostic challenge. ${ }^{4}$ Symptoms can develop slowly over the course of several weeks. ${ }^{5}$ The associated neurologic dysfunction that is evident can be highly variable. ${ }^{6}$ Much of the literature regarding this condition is in the form of case reports and retrospective data. Few prospective studies regarding CVT causes, diagnosis, or treatment are available.

The International Study on Cerebral Vein Thrombosis (ISCVT) is a multinational, multicenter prospective observational study whose objectives are to describe the long-term prognosis of CVT, to identify predictors of outcome, and to describe variability of CVT management and the associated risk factors and conditions. Four abstracts have been published on their web site regarding CVT (http://www.iscvt.org). ${ }^{2,7,8,9}$

This study group has reported that headache $(89 \%)$, paresis $(37 \%)$, generalized $(30 \%)$ or focal (20\%) seizures, papilledema (28\%), and mental status disorders (22\%) were the most frequent presenting symptoms and signs associated with the diagnosis of CVT. ${ }^{2}$ Twenty-three percent of patients had isolated intracranial hypertension. Nineteen percent had an admission Glasgow Coma Scale score of less than 14. Sixty-three percent had focal edema, an infarct (47\%), or hemorrhage (39\%) on the admission CT/MRI. The median delay from onset of symptoms to hospital admission was 4 days, very similar to the 5 days of headache symptoms experienced by this patient.

Many distinct underlying causes have been described for CVT. ${ }^{1-5,10-12}$ They include local factors (head injury, tumor, and craniotomy), infections (sepsis, sinusitis, and mastoiditis), coagulopathies (Protein C, S, and anti-thrombin III deficiencies, factor $\mathrm{V}$ Leiden, and antiphospholipid antibodies), systemic processes (pregnancy, systemic lupus erythematosus, and dehydration) and drug effects (oral contraceptives, hormone replacement therapy, androgens, and anabolic steroids.) CVT has also been described in newborns, especially those with prematurity, dehydration, or coagulopathies. ${ }^{13}$

The ISCVT group has reported that one (48\%) or multiple $(38 \%)$ predisposing conditions were identified in the majority $(86 \%)$ of their patients. ${ }^{9}$ The most frequent identified predisposing conditions were: oral contraceptives (46\%), prothrombotic conditions (27\%), pregnancy/puerperium (17\%), ear-nose-throat infections $(8 \%)$, central nervous system (CNS) infections (5\%), other infections (4\%), CNS disorders (5\%), cancer ( $8 \%$ ), vasculitis/antiphospholipid antibodies (8\%), and other systemic diseases (14\%). The cause was unknown in only $14 \%$ of patients. Subsequent evaluations over time may be able to later reveal a cause, as was the case with this patient. In hindsight, in this case it may have been of benefit to consider testing for hypercoagulable state at the time the thrombosis was diagnosed, because another definitive cause had not been determined.

The suspected diagnosis of CVT necessitates demonstration of the thrombus by neuroimaging. ${ }^{1}$ Noninvasive testing by MRI and now, less commonly, CT, has overtaken more invasive methods such as cerebral angiography. ${ }^{5}$

On noncontrast CT, the classic finding is the $\Delta$ sign, which is observed as a dense triangle within the superior sagittal sinus created by the hyperdense thrombus. ${ }^{1}$ This may not always be specific for CVT because a false-positive $\Delta$ sign may be seen in trauma patients as a result of an adjacent subdural hematoma. ${ }^{1}$ On contrast-enhanced CT, the reverse $\Delta$ sign (empty $\Delta$ triangle sign) can be observed in the superior sagittal sinus from enhancement of the dural leaves surrounding the comparatively less dense thrombosed sinus. This may not be typical, because it has been reported in only $20 \%$ of cases. ${ }^{13}$ The relative sensitivity and specificity of CT and MR studies has not been reported in the available literature. The false-negative rate for diagnosis by CT, estimated at $25 \%,{ }^{14}$ 
is considered high. MRV is now considered the investigation of choice. ${ }^{4,5,15-17}$

The inclusion criteria used by the ISCVT for the diagnosis of CVT by MRI include abnormal signal with in one of the sinuses, combined with absence of flow on MRA. ${ }^{2}$ By angiography, one would need to observe incomplete or absent filing or delayed emptying by one or more of the sinuses with signs of increased filling of collateral venous pathways.

Recommendations regarding the best treatment for patients with CVT have been difficult to make because of limited available evidence. The recently described dilemma of "nothing, heparin, or local thrombolysis" demonstrates that treatment choices are difficult in a disease process with variable presentation, causation and outcome. ${ }^{18,19}$ Intravenous heparin therapy in the acute phase is favored by most investigators and seems to be safe, even in patients with intracerebral hemorrhage in the area of a venous infarction. ${ }^{4,5,16,18,20-23}$ A recent metaanalysis concluded that anticoagulant treatment for cerebral sinus thrombosis seemed to be safe and was associated with a potentially important, although not statistically significant, reduction in the risk of death or dependancy. ${ }^{23}$ Use of thrombolytic therapy is generally reserved for patients who continue to deteriorate because of thrombosis extension despite optimal supportive management with dose-adjusted heparin. 5,18,19,22,24,25

The ISCVT group has reported on the types of treatment provided to the 624 patients in their study of care at 89 centers in 21 countries. $^{7}$ Either IV heparin or subcutaneous low-molecular-weight heparin was used in $82 \%$ of patients, reflecting a consensus between study participants. Considerable variations were found regarding use of local thrombolytics, steroids, other interventions to reduce intracranial pressure, and antiepileptics.

Mortality rates in CVT patients are now generally estimated to be in the $20 \%$ range. ${ }^{5,16,26}$ Short term prognosis was studied by the ISCVT. ${ }^{8}$ Multivariate predictors of death or dependency at discharge were low GCS score, advanced age, mental status disorders, hemiparesis, hemorrhage at admission CT, thrombosis of the deep venous system, and CNS disorder or cancer as a predisposing condition.

A long-term follow-up study of 77 patients with CVT over a mean of 78 months found that $86 \%$ had no neurologic sequelae. ${ }^{27}$ The frequency of long-standing epilepsy was low, indicating that most patients would not need long-term anticonvulsant therapy. A second CVT or another thrombolytic episode occurred in about $20 \%$ of their patients. Therefore, depending on the underlying cause of CVT, long-term anticoagulation might be required.

In conclusion, this patient's case of CVT demonstrates many prototypical features of this unusual process. Family physicians need to develop a broad differential diagnosis when evaluating patients with acute headache symptoms. Appropriately obtaining neuroimaging in those with signs and symptoms of focal neurological dysfunction is of the utmost importance.

\section{References}

1. Patel MR. Brain, venous sinus thrombosis, 2002 [cited 2003 May 17]. Available at: http://www. emedicine.com/radio/topic105.htm

2. Canhão P, Ferro JM, Bousser MG, Stam J, Barinagarrementeria F; the ISCVT collaborators. International Study on Cerebral Vein Thrombosis (ISCVT): baseline data [cited 2003 May 17]. Available at: http://www.iscrt.com/esc.htm

3. Grotta JC. Cerebral venous thrombosis-a new diagnostic challenge in travel medicine. J Travel Med 1996;1:137.

4. Fink JN, McAuley DL. Cerebral venous sinus thrombosis: a diagnostic challenge. Int Med J 2001; 31:384-90.

5. Kimber J. Cerebral venous sinus thrombosis. Q J Med 2002;95:137-42.

6. Murry BJ, Llinas R, Caplan LR, Scammell T, Pascual-Leone A. Cerebral deep venous thrombosis presenting as micrographia and hypophonia. Neurology 2000;54:751-3.

7. Ferro JM, Bousser MG, Barinagarrementeria F, Stam J, Canhão P; the ISCVT collaborators. Variation in management of acute cerebral vein and dural sinus thrombosis [cited 2003 May 17]. Available at: http://www.iscvt.com/esc.htm.

8. Ferro JM, Stam J, Bousser MG, Barinagarrementeria F, Canhão P; ISCVT investigators. The prognosis of acute cerebral vein and dural sinus thrombosis: ISCVT results [cited 2003 May 17]. Available at: http://www.iscvt.com/esc.htm.

9. Canhão $P$, Bousser $M G$, Barinagarrementeria $F$, Stam J, Ferro JM; the ISCVT collaborators. Predisposing conditions for cerebral vein and dural sinus thrombosis. Available at: http://www.iscvt.com/ index.htm [cited 2003 May 17].

10. Kriss TC, Kriss VM, Warf BC. Cavernous sinus thrombophlebitis: a case report. Neurosurgery 1996; $39: 385-9$. 
11. Lefebvre P, Lierneux B, Lenaerts L, et al. Cerebral venous thrombosis and procoagulant factors-a case study. Angiology 1998;49:563-71.

12. Saneto RP, Samples S, Kinkel RP. Case report: traumatic intracerebral thrombosis associated with an abnormal golf swing. Headache 2000;40:595-8.

13. Ramenghi LA, Gill BJ, Tanner SF, Martinez D, Arthur R, Levene MI. Cerebral venous thrombosis, intraventricular haemorrhage and white matter lesions in a preterm newborn with factor $\mathrm{v}$ (Leiden) mutation. Neuropediatrics 2002;33:97-9.

14. Ameri A, Bousser MG. Cerebral venous sinus thrombosis. Neuro Clin 1992;10:87-111.

15. Tsuruda JS, Shimakawa A, Pelc NJ, Saloner D. Dural sinus occlusion: evaluation with phase-sensitive gradient-echo MR imaging. AJNR Am J Neuroradiol 1991;12:481-8.

16. Cipri S, Gangemi A, Campolo C, Gafarelli F, Gambardella G. High-dose heparin plus warfarin administration in nontraumatic dural sinus thrombosis. A clinical and neuroradiological study. J Neurosurg Sci 1998;42:23-32.

17. Beeson MS, Vesco JA, Reilly BA, Little KJ. Dural sinus thrombosis. Am J Emerg Med 2002;20:568-9.

18. Benamer HT, Bone I. Cerebral venous thrombosis: anticoagulants or thrombolytic therapy. J Neurol Neurosurg Psychiatry 2000;69:427-30.
19. Bousser MG. Cerebral venous thrombosis: nothing, heparin, or local thrombosis? Stroke 1999;30:481-3.

20. Allroggen H, Abbott RJ. Cerebral venous sinus thrombosis. Postgrad Med J 2000;76:12-5.

21. Fink JN, McAuley DL. Safety of anticoagulation for cerebral venous thrombosis associated with intracerebral hematoma. Neurology 2001;57:1138-9.

22. Bousser MG. Cerebral venous thrombosis: diagnosis and management. J Neurol 2000;247:252-8.

23. Stam J, De Bruijn SF, De Veber G. Anticoagulation for cerebral sinus thrombosis. Cochrane Database Syst Rev 2002;(4):CD002005.

24. Niclot P, Bousser MG. Cerebral venous thrombosis. Curr Treat Options Neurol 2000;2:343-52.

25. Hsu FP, Kuether T, Nesbit G, Barwell SL. Endovascular therapy and neurological care: dural sinus thrombosis endovascular therapy. Crit Care Clin 1999;15:743-53.

26. de Bruijn S, de Haan R, Stam J. Clinical features and prognostic factors of cerebral venous sinus thrombosis in a prospective series of 59 patients. J Neurol Neurosurg Psychiat 2001;70:105-8.

27. Preter M, Tzourio C, Ameri A, Bousser MG. Longterm prognosis in cerebral venous thrombosis: follow-up of 77 patients. Stroke 1996;27:243-6. 